\title{
EvOLUCIÓN DE LA INDUSTRIA METALMECÁNICA EN JUÁREZ Y LA ADQUISICIÓN DE CONOCIMIENTO
}

\author{
EVOLUTION OF JUAREZ'S METALWORKING INDUSTRY AND \\ KNOWLEDGE ACQUISITION
}

Bertha Estrada Lázaro ${ }^{1}$ y Emmanuel Francisco García Uribe ${ }^{2}$

\section{RESUMEN}

4 ste artículo examina la evolución reciente en la industria metalmecánica en Juárez que se ha caracterizado por la aplicación del conocimiento en sus procesos productivos. Se profundiza en la identificación y caracterización de redes sociales empresariales desde un enfoque sociocéntrico. Los resultados que se presentan forman parte de un producto de investigación y revelan un sistema de interacciones entre las empresas participantes en relación a las fuentes de acceso y adquisición de conocimiento en un proceso de aprendizaje interactivo. El estudio identifica una diversidad de agentes líderes, entre los que se encuentran clientes, proveedores y empresas consolidadas en la localidad.

1 Universidad Vizcaya de las Américas. Campus Ciudad Juárez. Correo: b.estrada.dca. mx@gmail.com.

2 Universidad Autónoma de Ciudad Juárez. Correo: emmanuel.garcia@uacj.mx. 
Palabras clave: red interorganizacional, aprendizaje interactivo, comunidades de aprendizaje, grupos estratégicos, adquisición de conocimiento externo

\section{ABSTRACT}

This article examines the recent evolution of the Juarez metalworking industry, which has been characterized by the knowledge application in its production processes. It dives into the identification and characterization of business social networks from a sociocentric approach. The results presented form part of a research product that reveals a system of interactions between participating companies in relation to access sources and knowledge acquisition in an interactive learning process. The study identifies a diversity of leading agents, among which are clients, industry suppliers and local consolidated companies.

Key Words: interorganizational network, interactive learning, learning communities, strategic groups, acquisition of external knowledge

\section{INTRODUCCIÓN}

Las ciudades fronterizas al norte de México se han caracterizado por la atracción de inversión extranjera, la mano de obra y su dinámica poblacional. Su posición estratégica ha sido un elemento clave al encausar políticas sectoriales al proceso de industrialización y crecimiento económico y sus impactos sociales en diferentes sectores y actores de la región (Diario Oficial de la Federación [DOF], 1984). Dicha planeación para el caso Ciudad Juárez como la ciudad más poblada de Chihuahua es reflejada en cambios estructurales que han impactado la actividad económica y capacidad productiva de las empresas establecidas. Uno de los sectores predominantes es la industria metalmecánica con la producción 
de máquinas y equipos demandados por sectores económicos estratégicos del país.

La industria metalmecánica data desde los años de 1960 con la existencia de micro, pequeñas y medianas empresas (MIPYMES) como establecimientos de maquinados industriales. La importancia en el desarrollo de dicha industria contribuye de manera substancial a nivel nacional para una menor dependencia con respecto al exterior (Secretaría de Programación y Presupuesto [SPP] y Nacional Financiera [NAFINSA], 1982). Sin embargo, se considera un sector con poca capacidad para su integración en las cadenas globales de valor como proveedoras de bienes y servicios para la Industria Maquiladora de Exportación (IME) (Mendoza y Valenzuela, 2014).

A nivel local, también es considerado poco articulado con otras industrias, como la electrónica y automotriz (Ampudia y De Fuentes, 2009). Toda vez que posee un bajo nivel tecnológico y en los procesos de innovación y la inversión en tecnología (QuezadaTorres et al., 2018). Aunado a ello, los sectores de medio y alto nivel tecnológico tienen mayor presencia en la literatura científica que las de bajo nivel, como el caso de las empresas metalmecánicas (Dyer Singh y Hesterly, 2018; Tatarynowicz, Sytch y Gulati, 2016; Fahy, Easterby-Smith y Lervik, 2013; Bojica, Ruiz y Fuentes, 2012; Lawson, Petersen, Cousins y Handfield, 2009; Dyer, 2000). En adición, la trayectoria de dichos estudios a nivel local se han enfocado, entre otros, al aprendizaje tecnológico, capacidad tecnológica y derrama tecnológica (Mendoza y Valenzuela, 2014; Vera-Cruz y Dutrénit, 2009), a partir de la influencia de la $\mathrm{IME}^{3}$ con empresas de maquinados. En otras palabras, el aprendizaje y capacidad de absorción a partir de los clientes y, en menor medida, desde la adquisición de conocimiento externo entre pares y proveedores del sector metalmecánico.

3 La IME es un tipo de industria que por decreto se le permitía su instalación en México y la importación temporal de maquinaria e insumos sin pagar impuestos, y donde su producción fuera destinada a la exportación, bajo un proceso de transformación, elaboración, o bien, reparación de mercancías, tal como se establece en los pronunciamientos del Diario Oficial de la Federación a partir de 1980 y con sus posteriores modificaciones (DOF, 1983; 1998; 2006). 
Estos antecedentes permiten examinar con quién y cómo se genera ese aprendizaje desde el enfoque del comportamiento interorganizacional (nivel micro). Asimismo, cómo el sector se adapta e integra con el entorno regional (nivel meso), toda vez que, en la localidad, la apertura de nuevos establecimientos es continuo, a pesar de las limitaciones estructurales en capacidades y recursos. Por lo tanto, el abortar el tema desde la interacción pretende la obtención de información relevante sobre la vinculación en redes, como una alternativa a las fuentes de competitividad en las empresas y con la ausencia de estudios relacionados a comunidades de aprendizaje.

Ante este planteamiento, el acceso y adquisición de conocimientos especializados en la fuerza laboral es considerado un recurso clave entre las organizaciones; en un contexto nacional, brinda competitividad en las economías de las regiones en desarrollo (Drucker, 2003; 1999; Dyer, 2000); toda vez que el valor del mismo dependerá de la información que se posee y es determinante en la obtención de ventajas tecnológicas y organizativas (Hayek, 1945; 1937). En este proceso, el conocimiento y el aprendizaje se consideran capacidades dinámicas cuando se generan interacciones sociales entre individuos y organizaciones (Brix, 2017; Nonaka, Toyama y Konno, 2000), desde el enfoque de explorar y explotar conocimiento al institucionalizarlo.

Por lo tanto, este estudio tiene como objetivo examinar la evolución en las empresas metalmecánicas y el desarrollo de sus capacidades desde el paradigma del conocimiento y aprendizaje interactivo en comunidades y grupos estratégicos en un entorno meso y micro en Ciudad Juárez. Este documento integra y analiza evidencia científica; distingue los acontecimientos y determinantes a través del tiempo del sector; describe su composición con datos de fuentes oficiales como el Instituto Nacional de Estadística y Geografía (INEGI) y el DOF; e identifica, caracteriza y contrasta con los resultados de la investigación sobre la dinámica relacional en la adquisición de conocimiento por medio de una retícula.

Estos resultados se derivan de un estudio (Estrada, 2020) de investigación no experimental, con alcance descriptivo, explorato- 
rio y explicativo de análisis de redes. La recolección de datos primarios se llevó a cabo con la participación de cuarenta empresas del sector, con el desarrollo y aplicación de un cuestionario con preguntas semiestructuradas, administrado a personal de mando medio y superior a nivel de unidad de negocio que evidenciara la interacción con otra u otras empresas y/o instituciones (clientes, proveedores, competidores e instituciones), con información para el periodo de 2017-2018. Lo anterior, para aproximarse a la realidad en la que prevalece el sector durante la segunda década del siglo XXI.

La estructura del presente documento inicia con el desarrollo de la industrialización fronteriza, la maquiladora y el sector metalmecánico; seguido de apartados relacionados con el aprendizaje interactivo en redes empresariales y grupos estratégicos para la adquisición de conocimiento y capacidades en el entorno regional, y finaliza con la discusión de la investigación y conclusiones.

\section{EL DESARROLLO DE LA INDUSTRIALIZACIÓN FRONTERIZA EN JUÁREZ}

Las estrategias trazadas de políticas, programas y proyectos a nivel federal parten de la dinámica en la región que data desde mediados del siglo XIX -zonas y perímetros libres- con asentamientos al norte de México (Timmons, 1990; Mendoza, 1982) y a partir del siglo xx con atracción de mano de obra hacia los Estados Unidos y la Crisis económica de 1929, ante la posibilidad de mejorar los ingresos (Cerutti, 2013; Mendoza, 1982); la apertura comercial Zona Libre en 1939- para abastecer a la localidad fronteriza de bienes (Fuentes y Fuentes, 2004); además del Acuerdo Internacional sobre Trabajadores Migratorios -Programa de Braceros de 1951 a 1964- y la política de irrigación del cultivo del algodón -1950-1970- (Cerutti, 2013). Seguido de proyectos agroindustriales, actividad comercial, de servicios e industrialización fronteriza con plantas ensambladoras (Cerutti, 2013; Vera-Cruz y Dutrénit, 2009: Vera-Cruz y Gil, 2006; Vera-Cruz y Dutrénit, 2004; Mendoza, 1982; Jiménez y Sánchez, 2015; Villavicencio, 2006). 
El declive de algunas actividades y programas, como el algodón y el Programa Braceros, y el desempleo local trajo consigo la implementación del Programa Nacional Fronterizo (PRONAF) entre los años de 1961 a 1965, basado en la promoción de acciones de infraestructura, centros comerciales, generación de empleos, con ello acrecentó actividades de comercio y turismo (Calderón y Arenaza, 2015; Mendoza, 1982); a su vez, el Programa de la Industria Maquiladora (Fuentes y Fuentes, 2004). Las empresas de inversión extranjera instaladas en la región se considerarían bajo el concepto desarrollado de primera generación, con base en las capacidades que predominaban en cuanto a su integración vertical, tecnología, innovación, organización, autonomía en la toma de decisiones y capital humano (Carrillo y Gomis, 2005).

Desde la primera etapa de plantas ensambladoras en la zona de libre comercio -1965-1970- (Fuentes y Fuentes, 2004; Vera-Cruz y Gil, 2006) y la inversión en naves y parques industriales (Calderón y Arenaza, 2015); también con iniciativas privadas que emergieron en el año de 1973 con proyectos de desarrollo estratégico a nivel estatal (Desarrollo Económico del Estado de Chihuahua, A. C. [DESEC], 2018), promoviendo una economía del sector primario a una de la transformación (Casalet y González, 2006). Otros acontecimientos se pueden referenciar posteriores a la década de 1970 (Figura 1) como son las devaluaciones económicas -1976, 1982- y con ello la inestabilidad en el mercado cambiario, costos en insumos y bienes importados, y la devaluación de la moneda (DOF, 1984); políticas de industrialización y comercio exterior, por ejemplo, el Programa de Desarrollo Fronterizo (Fuentes y Fuentes, 2004), la adhesión al Acuerdo General Sobre Aranceles Aduaneros y Comercio (Calderón y Arenaza, 2015; DOF, 1986); y para los años de 1990, con el Tratado de Libre Comercio para América del Norte (TLCAN) (DOF, 1993), propiciando un mayor intercambio comercial con el establecimiento de la zona de libre comercio (Calderón y Arenaza, 2015; DOF, 1993). Para esa década la generación de empleos por parte de la IME se había consolidado en la región (Calderón y Arenaza, 2015; Dutrénit y Vera-Cruz, 2009), pasando de una economía basada en el sector primario a una industrializa- 
da (Casalet y González, 2006). Aunado a ello, plantas maquiladoras de segunda generación establecidas con nuevo tipo de tecnología y procesos automatizados, es decir, mayor complejidad organizacional y con personal altamente calificado (Carrillo y Matus, 2020; Carrillo y Gomis 2005).

Los efectos de la crisis de la década de 1980 y las políticas de comercio exterior implementadas en la década de 1990, además de las implicaciones para ciertos sectores, llevan a cabo la generación de estudios estratégicos sectoriales en el estado de Chihuahua, con el diagnóstico de la estructura económica para el desarrollo económico -Programa Chihuahua Siglo XXI- y el análisis del ambiente industrial predominante en la región, por ejemplo, evidenciando clústeres potenciales e iniciativas de centros de investigación (Ávila, 2004; Morales, 1999; Centro de Estudios Estratégicos ITESM, 1993; Casalet y González, 2006). En adición, a mediados de la última década del siglo xx, autores como Carrillo y colaboradores (Carrillo y Matus, 2020; Carrillo y Gomis, 2005) identificaban una tercera generación de la IME caracterizada por la generación de conocimiento a partir de actividades de diseño y prototipos por medio de la Investigación y Desarrollo (I+D).

La situación durante la primera década del siglo xxi para Juárez, aunado a la problemática de inseguridad, permite integrar programas emergentes bajo el planteamiento de una región atractiva, con eventos como Juárez Competitiva y el programa Todos Somos Juárez, Reconstruyamos la Ciudad (Secretaría de Economía [SE], 2012). Ante dichas problemáticas sociales, en el contexto de desarrollo económico en la región sobre Inversión Extranjera Directa (IED) se define una cuarta generación de maquilas, con capacidades asociadas a las tecnologías de la información (Carrillo y Matus, 2020). En la segunda década se estableció un trato diferenciado para la franja fronteriza con la homologación del Impuesto al Valor Agregado (IVA), con acciones para fomentar mayor infraestructura en telecomunicaciones y transporte (Fuentes, Ruiz, González y Brugués, 2016).

Los antecedentes presenciados a nivel político, social y en la activación económica de la frontera norte de México, aunadas a 
la apertura comercial, de servicios, en términos fiscales y en el desarrollo de la industrialización fronteriza para el caso de Juárez (Figura 1) determinan el contexto de las actuales empresas del sector de estudio. Otros acontecimientos más enfocados al sector metalmecánica serán comentados durante el desarrollo del documento.

\section{LA INDUSTRIA MAQUILADORA Y EL SECTOR METALMECÁNICO EN JUÁREZ}

La actividad económica de las regiones se diferencia por el tipo de bienes o servicios predominantes. En el caso de Juárez, presenta cambios en la estructura económica durante la segunda mitad del siglo xx, es decir, de actividades agroindustriales a la presencia de empresas bajo el régimen de maquiladora con inversión extranjera directa y su influencia en las Pequeñas y Medianas Empresas (PYMES) de la localidad, a partir de la premisa que, el alojar este tipo de industrias, permiten beneficios y el incremento de las capacidades en las regiones y países (Contreras et al., 2012).

La industria metalmecánica forma parte de sectores tradicionales, estratégicos y dinámicos, y de las estrategias a nivel nacional en la promoción de cadenas de valor y desarrollo de capacidades productivas; a nivel micro, se promueven estrategias de capacitación y certificación que contribuyan al desarrollo de capital humano, procesos y productos (DOF, 2015). Este tipo de empresas como unidades económicas son clasificadas en el Ramo 31-33, donde predominan plantas, fábricas y talleres, entre otros, dedicados grosso modo a la transformación, ensamble, reconstrucción y al acabado de productos manufacturados (INEGI, 2018a; INEGI, 2018b).

El sector de la industria metalmecánica tiene un rol relevante a nivel nacional con la producción de máquinas y equipos para la IME. La conforman diferentes subsectores y clasificaciones, entre ellas: Industrias metálicas básicas, Industria básica del hierro y del acero, Fabricación de productos de hierro y acero, Industria básica del aluminio, Industrias de metales no ferrosos, excepto aluminio; 
EVoluCiÓn DE LA INDUSTRIA METALMECÁNiCA EN JUÁREZ...

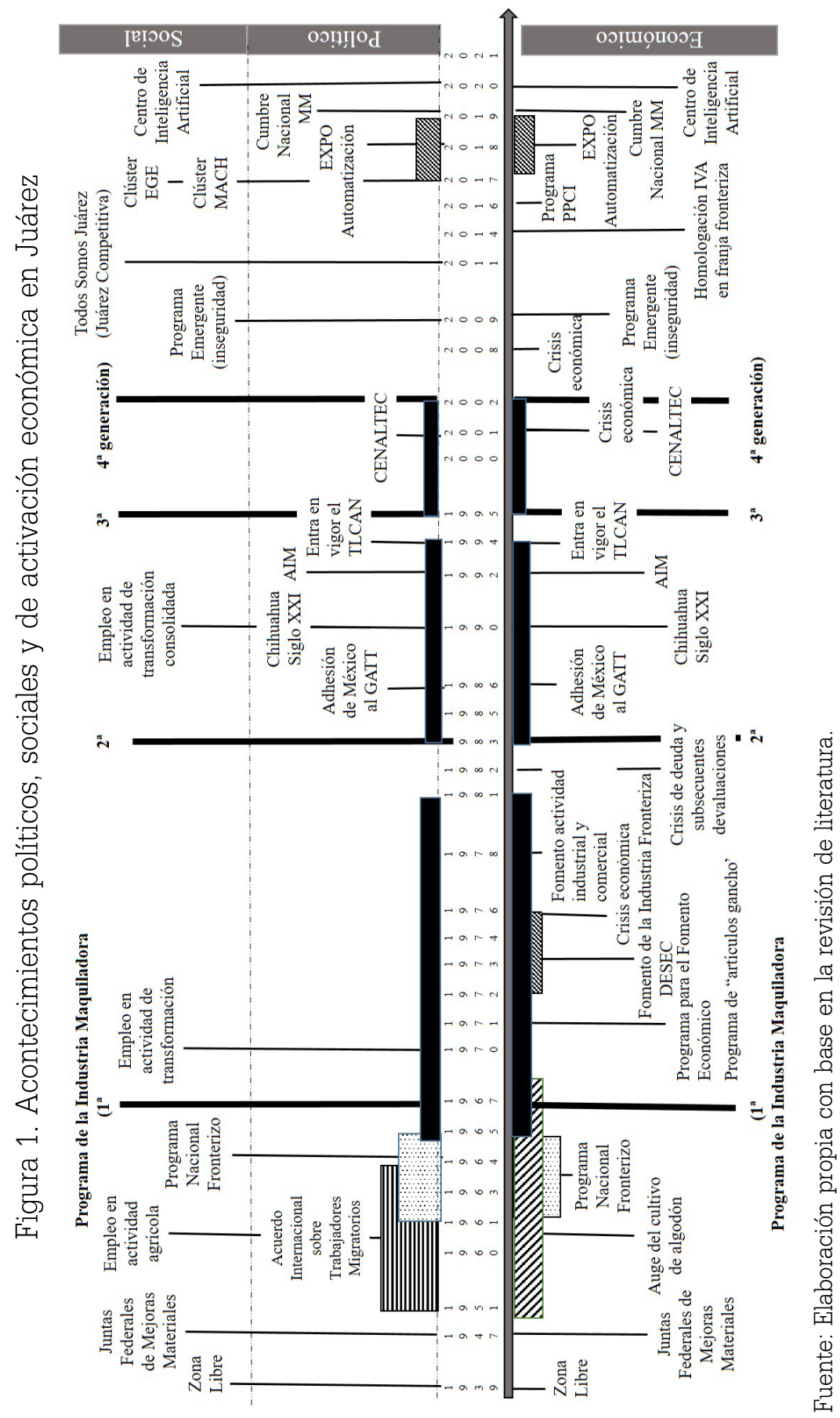


Moldeo por fundición de piezas metálicas, Fabricación de productos metálicos, Fabricación de maquinaria y equipo, Fabricación de equipo de computación, comunicación, medición y de otros equipos, componentes y accesorios electrónicos; Fabricación de accesorios, aparatos eléctricos y equipo de generación de energía eléctrica; Fabricación de equipo de transporte, Fabricación de muebles, colchones y persianas; y Otras industrias manufactureras (INEGI, 2018a). Sin embargo, este estudio, se enfoca a las actividades económicas relacionadas con subsectores de encadenamientos de proveeduría nacional en la IME, es decir, establecimientos del subsector de Fabricación de productos metálicos (332) y Fabricación de maquinaria y equipo (333) donde predominan las MIPYMES y PYMES en la región.

Se caracterizan por la diversificación de productos y servicios derivados de demandas del mercado y complementando recursos y conocimiento con otros giros empresariales, por ejemplo, servicios.

Considerando lo anterior, la industria del sector metalmecánico integra al sector de "maquinados industriales de precisión [...] conformado por las empresas industriales que diseñan y fabrican piezas de precisión" (Vera-Cruz y Dutrénit, 2004, p. 70) con equipos como fresadoras, tornos, centros de maquinado, entre otros a diferentes niveles de complejidad y nivel tecnológico (Vera-Cruz y Dutrénit, 2004).

De acuerdo con el Directorio Estadístico Nacional de Unidades Económicas (DENUE) (INEGI-DENUE, 2018a), sobresalen (Tabla 1) un total de 150 establecimientos en el sector de la industria metalmecánica para el año 2021, relacionadas con los encadenamientos de proveeduría nacional en Juárez. En este dato se incluyen empresas nacionales y de Inversión Extranjera Directa (IED) de diferentes estratos como son MIPYMES y grandes empresas. A nivel nacional representan el $1.27 \%$ y $1.37 \%$; y el $39.43 \%$ y $41.09 \%$ a nivel estatal durante los años 2018 y 2021, respectivamente. Sin embargo, estos números no reflejan las empresas constituidas bajo el régimen de personas físicas en la región dedicadas a brindar servicios de proveeduría a la IME. 
Evolución DE LA industria MetalmecÁNiCA EN JuÁREZ...

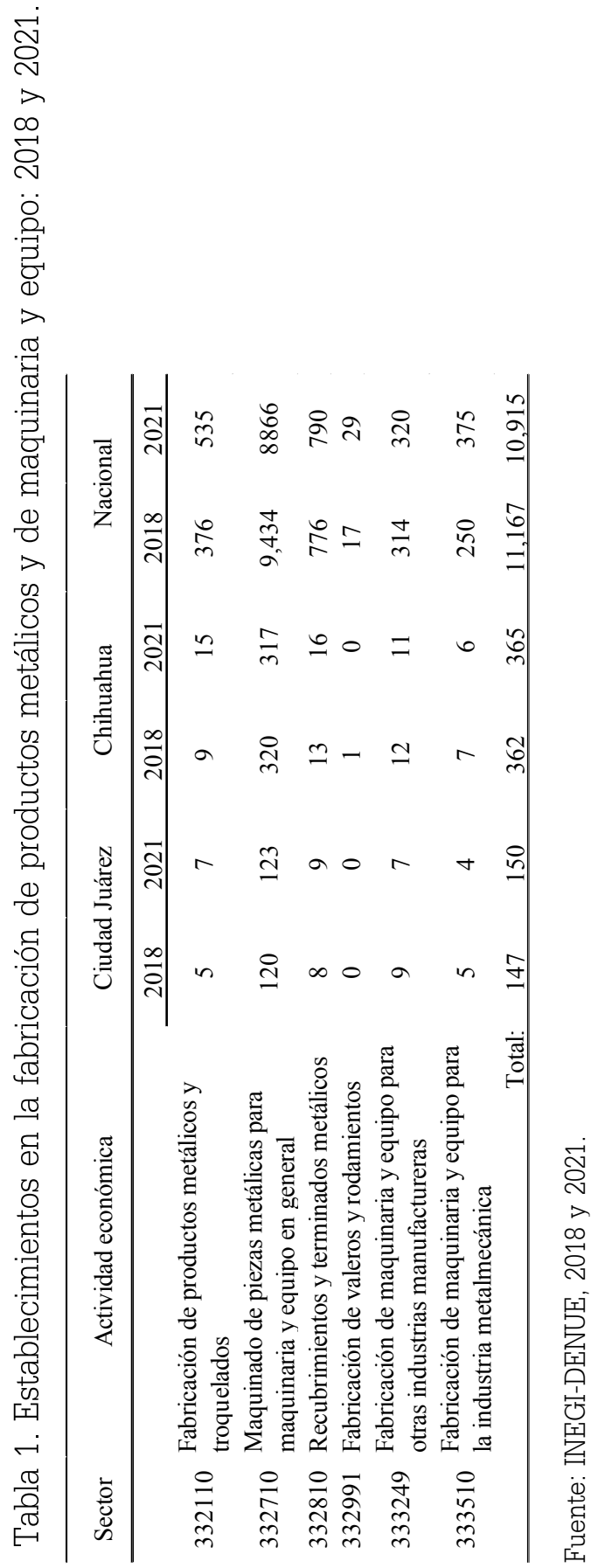




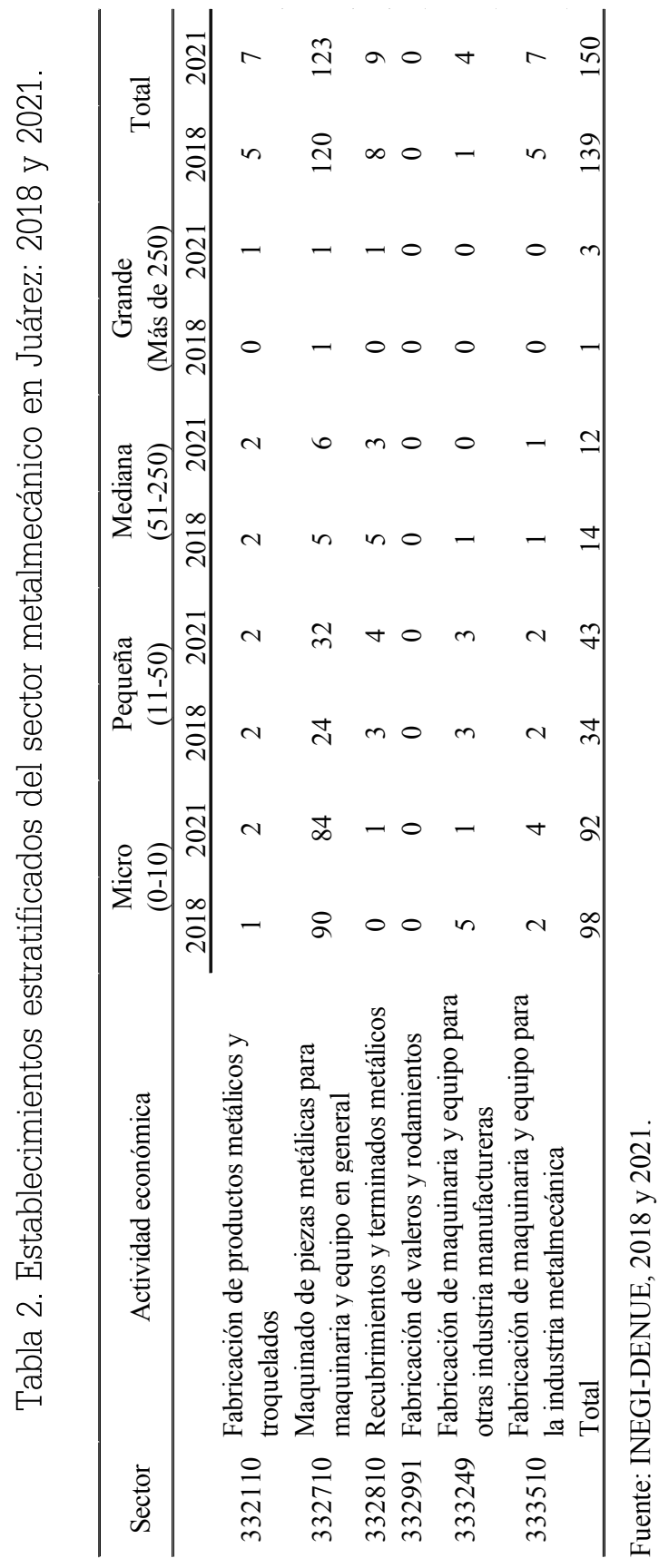


En la Tabla 2 se muestra la composición de empresas metalmecánicas por su tamaño y número de trabajadores, donde el $66.67 \%$ y $61.33 \%$ la constituyen microempresas; el $23.13 \%$ y $28.66 \%$ son pequeñas; un $9.52 \%$ y $8 \%$ son medianas; y el $1 \%$ y $2 \%$ son grandes empresas durante el 2018 y para el 2021, respectivamente.

Por lo descrito hasta ahora, el sector de la industria metalmecánica ha sido estudiado a nivel nacional (Cornejo et al., 2011; SPP y NAFINSA, 1982; Katz, 1982) y local (Argueta y Jiménez, 2017; Jiménez, Argueta y Leiner, 2017; Jiménez y Sánchez, 2015; Arellano, 2013; Dutrénit y Vera-Cruz, 2009; Ampudia y De Fuentes, 2009; Donjuan, 2008; Casalet y González, 2006; Vera-Cruz y Gil, 2006; Vera-Cruz y Dutrénit, 2004). En ellas, se menciona como una industria incipiente desde los años de 1980 por las prácticas proteccionistas y con un avance tecnológico parcial (Katz, 1982). No obstante, la diversificación de servicios de algunas empresas en la localidad permiten, para efectos de este estudio, considerar a una empresa metalmecánica a todas aquellas que brinden servicios y productos de maquinados, automatización y manufactura, diseño mecánico, software y programación, integración de equipo industrial, pintura electrostática, estampado, tecnología de plásticos, corte láser, galvanoplastia y soldadura industrial (Estrada, 2020).

\section{INDUSTRIA METALMECÁNICA: APRENDIZAJE INTERACTIVO Y CAPACIDADES}

El sector de la industria metalmecánica en Juárez, localizada en zona fronteriza, es abordada a partir del impacto de las empresas multinacionales que han tenido a través del tiempo, toda vez que ha permitido a las empresas apropiarse y desarrollar capacidades distintivas en desarrollo de capacidades gerenciales y talento humano, así como en inversión en recursos e infraestructura; a diferencia de otras regiones del país, reflejado esto como ventajas competitivas sostenibles. También, el sector de la industria metalmecánica en Juárez ha sido estudiado desde las multinaciona- 
les para entender los entornos interno y externo de las empresas actuales. En este sentido, las empresas establecidas en Ciudad Juárez para el siglo XXI con base en el contexto de análisis de zona fronteriza ha permitido a las mismas apropiarse y desarrollar capacidades distintivas con respecto a otras regiones del país; en términos de gestión del conocimiento, la inversión de recursos, el tipo de infraestructura y el desarrollo de capacidades gerenciales y talento humano. Ello, derivado de los diversos niveles de formalización de las relaciones con la IME, en búsqueda de ser competitivos, tener acceso a información y por medio de un aprendizaje interactivo (Vázquez, 1999); en otras palabras, desarrollado en un contexto social, a partir del acercamiento de las empresas que aprenden (metalmecánicas) con empresas maestras (IME) para la creación de valor (Lane y Lubatkin, 1998). Además, la vinculación durante las diferentes generaciones de maquiladoras (Carrillo y Matus, 2020: Carrillo y Gomis, 2005), los cambios estructurales en las redes de proveduría de la IME (Villavicencio, 2006), así como, incentivos federales e iniciativas de particulares en colaboración con el gobierno y la academia, que han impactado a dicho sector.

En complemento con la Figura 1, en la Figura 2 se visualiza el proceso evolutivo del sector desde el enfoque del conocimiento y capacidades. Al respecto, durante los años de 1940 a 1960, el sector contaba con una estructura artesanal y se enfocaban a proveer al sector agroindustrial (Vera-Cruz y Gil, 2006; Vera-Cruz y Dutrénit, 2004) como el algodón -en 1950 a 1960, a nivel estatal, se contribuía entre el $10 \%$ a $12 \%$ del total de la producción nacional- (Cerutti, 2013). Posteriormente, a mediados de la década de 1960, se crean oportunidades de nuevo mercado con la IME con las primeras generaciones de maquilas, caracterizadas por tener baja tecnología, ensamble simple y una desvinculación con la industria nacional, aunado a que los proveedores estaban localizados fuera de México (Carrillo y Gomis, 2005; Vera-Cruz y Dutrénit, 2004). Ello representó que durante la siguiente década, de 1970, se contara con cinco empresas en la localidad de talleres de maquinados y la necesidad por parte de la IME de realizar compras 
EVOLUCiÓN DE LA INDUSTRIA METALMECÁNICA EN JUÁREZ...

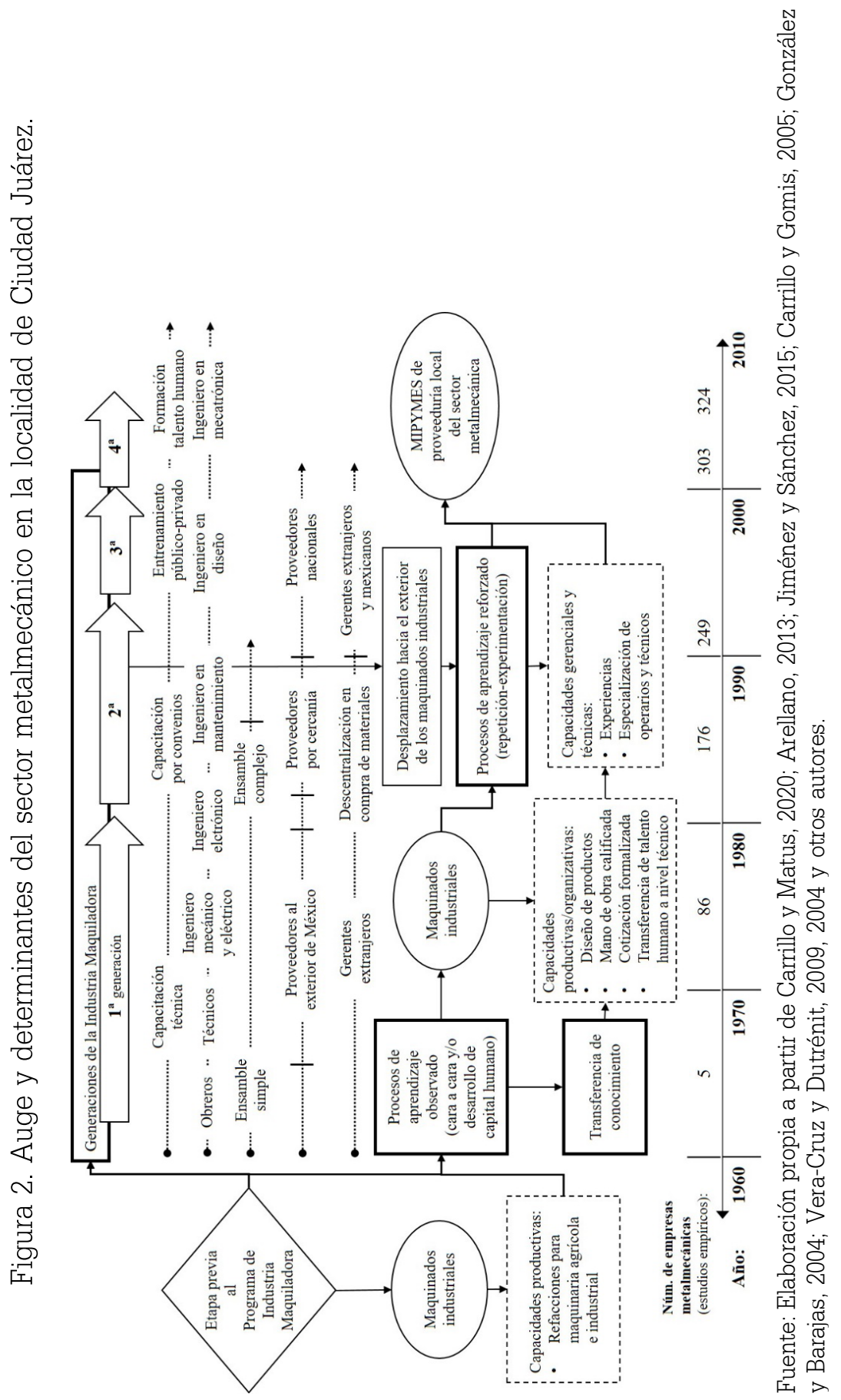


indirectas de partes y refacciones (Arellano, 2013; Vera-Cruz-Gil, 2006) en la región.

La ausencia de capacidades locales en el momento permitió constituir vínculos público-privados en la capacitación de operarios y técnicos en máquinas y herramientas de egresados de Educación Media Superior Técnica. Un ejemplo de ello es el establecimiento del sector automotriz en México (Katz, 1982; Jiménez y Sánchez, 2015; Villavicencio, 2006) y sus contribuciones de manera directa e indirecta en procesos organizativos y de manufactura en las actividades productivas y gerenciales en las empresas locales (Contreras et al., 2012). Además, en "el desarrollo de instituciones de capacitación y educación, la conformación gradual de un ambiente binacional, el surgimiento y consolidación de asociaciones empresariales orientadas a la problemática local" (Vera-Cruz y Dutrénit, 2004, p. 2). Con todo lo anterior, las competencias desarrolladas permiten un incremento en el número de establecimientos, al generar y acumular conocimiento en la localidad, con la vinculación y la transferencia de conocimientos técnicos y de gestión (González y Barajas, 2004; Vera-Cruz y Dutrénit, 2004). Ejemplo de ello es la generación de vínculos sociales y profesionales por parte de empresarios y emprendedores exempleados de la IME que proveían trabajos especializados para dar respuesta inmediata a problemas técnicos a nivel local (Contreras et al. 2012).

A partir de la década de 1980 el número de empresas instaladas se iba incrementando (Figura 2), a pesar de que las prácticas de la IME, a mediados de dicha década, se caracterizaban por el abastecimiento de insumos al exterior del país (González y Barajas, 2004).

Sin embargo, se propicia el desarrollo de proveedores cercanos de insumos y servicios, tanto directos e indirectos (Carrillo y Gomis, 2005). Para los últimos años, un mayor número de empresas se establecieron con el objetivo de incrementar el volumen y la diversificación de la producción; esto implicaba mayor capacidad productiva, trasladarse de un ensamble simple o manual a uno más automatizado y requerir personal más calificado, entre ellos, técnicos e ingenieros con habilidades gerenciales (Villavicencio, 
2006; Carrillo y Gomis, 2005). Al mismo tiempo, en el ambiente de la oferta educativa se distinguía por las carreras técnicas que permitían la formación de un mayor número de ingenieros acorde a las necesidades de la IME derivado de convenios (Dutrénit y Vera-Cruz, 2009; Vera-Cruz y Dutrénit, 2004).

A mediados de la década de 1990, se caracterizó por el establecimiento de centros técnicos en la región por las EMN como Delphi y Valeo (De los Santos, Carrillo, Plascencia, Villavicencio y Esparza, 2017; Villavicencio, 2006). Aunado a ello, también se generan cambios estructurales al incluir los departamentos de ingeniería y desarrollo, que implicó mayor atención al aprendizaje en capacidades tecnológicas (Arellano, 2013; Contreras et al., 2012; Carrillo y Gomis, 2004; González y Barajas, 2004) y nueva diversificación de conocimiento en la localidad (Vera-Cruz y Dutrénit, 2009; Carrillo y Gomis, 2004). La presencia de este tipo de multinacionales de tercera generación permite la integración vertical con proveedores nacionales, para suministrar materiales de empaque y servicios indirectos por parte de empresas de maquinado (Carrillo y Gomis, 2005; Dutrénit y Vera-Cruz, 2009; Vera-Cruz y Dutrénit, 2004), como es la demanda y la evolución en el número de establecimientos a principios de la década del 2000, 303 en total (Vera-Cruz y Dutrénit, 2004). A partir de este escenario, se emprendieron acciones asociativas para empresas de proveeduría nacional con la creación de la Asociación de Industrias del Maquinado (AIM) (Vera-Cruz y Gil, 2006).

Para el año 2002, los estudios sobre la proveeduría en Juárez (Carrillo y Gomis, 2004), evidencia que un 12.8 \%, en términos relativos, contaba con proveedores nacionales en el área de estructuras metálicas; y un promedio del $4.8 \%$ proveniente de Juárez, de las ensambladoras de la localidad (El Colegio de la Frontera Norte [El COLEF], 2002), predominando en ese periodo una manufactura de mayor complejidad en la IME (Villavicencio, 2006).

En términos de aprendizaje colectivo, redes de proveeduría (Contreras et al., 2012) y educación continua, se derivó la conjunción de esfuerzos públicos y privados. Por ejemplo, con la iniciativa de la empresa Royal Philips Electronics (Dutrénit y Vera-Cruz, 
2009; Hualde, 2006) en el año 2001, para la formación de talento humano en habilidades y capacidades técnicas, entre ellas la industria metalmecánica, por medio del Centro de Entrenamiento en Alta Tecnología (CENALTEC), en coordinación con el Instituto de Apoyo al Desarrollo Tecnológico (INADET); a nivel federal con la Dirección General de Centros de Formación para el Trabajo (DGCFT) y la Secretaría de Educación Pública (SEP); y a nivel estatal, la Secretaría de Innovación y Desarrollo Económico (SIDE) y el Gobierno del Estado de Chihuahua (Instituto de Apoyo al Desarrollo Tecnológico [INADET)], 2018) para emprender el proyecto con los programas de entrenamiento, infraestructura, personal técnico y equipo necesario. Sin embargo, la crisis económica dificultó el desarrollo y potencial del proyecto. Otras acciones son evidentes en la década durante el 2001 y 2007 por parte de convenios de Delphi e instituciones de educación en el área de ingeniería mecánica y mecatrónica (Dutrénit y Vera-Cruz, 2009).

La constitución de asociaciones industriales, así como de clústeres que emergen en el entorno regional, son percibidas como capacidades para generar experiencias en comunidades de aprendizaje colectivo e interactivo, redes de transferencia de conocimiento y nuevos recursos tecnológicos en el sector de la industria metalmecánica (Contreras et al., 2012; Cornejo et al., 2011). Dentro de estas prácticas, en la última década del 2010, se conforma en el estado el Clúster de Empresas Globales Emergentes (Clúster EGE) con inicio de operaciones en el año 2017 (Centro de Competitividad Chihuahua, 2017) y constituido con empresas locales y nacionales para la promoción de cadenas de proveeduría local en un contexto de cadenas globales de suministro (Clúster EGE, 2019).

Un segundo caso en Juárez es la conformación del Clúster de Manufactura Avanzada de Chihuahua (Clúster MACH, 2020). Esta asociación integra doce empresas de capital nacional y parte de la naturaleza del concepto de clúster industrial (Clúster MACH, 2008), a partir de un sistema local constituido por diversas empresas de una o varias industrias que interactúan entre sí y con el entorno que las rodea (Brenner, 2000). Las gestiones que realiza se enfocan en la capacitación, asesoría, desarrollo de negocios y 
procuración de fondos de empresas de la industria metalmecánica; (Clúster MACH, 2018). Al cierre del año 2019, reportaba una capacidad instalada de \$20.6 millones de dólares, con un área de operación de 453,65 pies cuadrados y $\$ 29.45$ millones de dólares en ventas anuales y con una generación de empleos de 662 personas (Clúster MACH, 2020).

Existen otras instituciones en la región que promueven la competitividad de la industria metalmecánica a nivel local que no son comentados en este documento, entre ellos están: la Cadena de Proveedores de la Industria en México (CAPIM); la Asociación Mexicana de Fabricación de Moldes y Troqueles (AMMMT); el Clúster de Moldes y Troqueles; el Centro de Inteligencia Artificial (IACenter) y el Centro de Investigación en Ciencia y Tecnología Aplicada (CICTA).

Por todo lo comentado, a partir de diversas décadas en Juárez, se ha generado la demanda de personal especializado y empresas metalmecánicas con la creación y, a su vez, el desarrollo de capacidades productivas de proveeduría local. Para el año 2021 se cuentan con 365 empresas a nivel estatal y 150 en la región (INEGI, 2021), sin considerar las personas físicas no registradas en esta fuente oficial. Este desarrollo se llevó a cabo por medio de un proceso de aprendizaje interactivo -observación y reforzamientocon la IME, derivado de la movilidad laboral y experiencias previas y la creación de empresas de proveeduría a nivel local (Arellano, 2013; Vera-Cruz y Dutrénit, 2009). En este proceso de transferencia de conocimiento de capacidades gerenciales y organizativas se encuentran la adquisición de conocimiento tácito, prácticas empresariales, visión empresarial y acceso a mercados; además de tecnología de equipo, diseños organizacionales con el uso de sistemas operativos y procesos de producción en el diseño de piezas y moldes (Vera-Cruz y Dutrénit, 2009). En otras palabras, a partir de la experiencia, capacitación y redes sociales surge la creación de dichas empresas (Contreras et al., 2012; Vera-Cruz y Gil, 2006) de manera gradual en el contexto local. 


\section{ADQUISICIÓN, APRENDIZAJE Y CAPACIDADES EN LAS RELACIONES INTERORGANIZACIONALES}

El conocimiento es un recurso clave para la estrategia y la competitividad en las organizaciones y desde el paradigma relacional implica prácticas de cooperación y competencia en la creación y captura de valor, y dependerá de cómo se realiza la gestión para el desarrollo de capacidades a nivel individual y colectivo (Dyer et al., 2018; Menolli, Reinhr y Malucelli, 2013), con la finalidad de crear, adquirir, integrar, implantar y distribuir conocimiento (Menolli et al., 2013).

El adquirir conocimiento y el aprendizaje de nuevas capacidades se considera un proceso dinámico, toda vez que se forja a partir de interacciones sociales entre individuos y organizaciones a diferentes niveles (Brix, 2017; Nonaka, Toyama y Konno, 2000; Chandler, 1992). La adquisición de conocimiento es un proceso de exploración, como un mecanismo para generar conocimiento (Grant, 2010). Mientras que la adquisición de conocimiento externo es la capacidad para identificar conocimiento distante fuera de los límites de la organización (Abarca y Barreto, 2021; Grant, 2010). A nivel gerencial estas funciones hacen referencia a una meta-capacidad, ante la necesidad de generar un ambiente de aprendizaje de nuevos procesos y habilidades, compartir y aprovechar dicho conocimiento, además, de una comunicación abierta, flexible y adaptable en la estructura organizativa para adquirir conocimiento desde fuera de la organización (Dawson, 2005).

En este sentido, una de las teorías que da importancia a un aprendizaje continuo y toma de decisiones basadas en el entorno externo es la teoría de los recursos y capacidades (Chandler, 1992), a partir del análisis de los recursos y evaluación de las capacidades y competencias centrales con que cuenta la empresa para el crecimiento estratégico, al integrarse con otras empresas y poder potencializar recursos desde el exterior (Chandler, 1992; Grant, 1991). Se interpreta como un proceso intraorganizacional para adaptarse a los cambios externos desde la gestión de conoci- 
miento y la perspectiva relacional y redes (Dyer et al., 2018; Dyer y Singh, 1998; Grant, 1991).

Esto emerge de la generación de vínculos por la interdependencia y confianza entre organizaciones con atributos diferentes y su funcionalidad por medio de rutinas asociativas, en el intercambio y transferencia de conocimiento, así como de programas conjuntos de cooperación, al involucrar y complementar recursos y capacidades, cuando se comparte conocimiento en una relación a nivel interorganizacional como práctica en la adquisición de conocimiento (Gibb, Sune y Albers, 2017; Nodari, Oliveira y Gastaud, 2016; Bojica et al., 2012; Goerzen, 2007; Lane y Lubatkin, 1998; Aiken y Hage, 1968).

En las relaciones interorganizacionales se establece un aprendizaje interactivo para adquirir nuevo conocimiento externo; este se desarrolla entre dos tipos de empresas en un contexto social, empresas que aprenden -estudiantes- y su relación con empresas maestras con el fin de crear valor estratégico (Lane y Lubatkin, 1998). La información recibida por la empresa que aprende se adquiere por medio de conocimiento tácito con la interacción cara a cara o un tipo de conversación al comparar experiencias y conectándolo (Lane y Lubatkin, 1998; Dyer y Singh, 1998) con conocimiento existente, así como transformándolo en nuevo, en función de las capacidades que la empresa posea (Lane y Lubatkin, 1998) y las habilidades para alcanzarlo. Otra teoría en el tema de las redes interorganizacionales (Van de Ven, 1976) es la del aprendizaje organizacional (Watkins y Bell, 2002; Knight, 2002) para la capacidad de absorción, al compartir (Nodari et al., 2016) y adquirir conocimiento.

La capacidad puede ser definida a partir de la funcionalidad del concepto. La capacidad organizacional se considera una habilidad para la realización de tareas con la utilización de recursos organizacionales para el logro de un resultado final (Manuj y Yazdanparast, 2013). Las capacidades tecnológicas son integradas por un conjunto de habilidades para el uso eficiente del "conocimiento tecnológico adquirido; para asimilar, utilizar, adaptar y cambiar tecnologías existentes, así como las habilidades para crear nuevas 
tecnologías y desarrollar nuevos productos y procesos" (Mendoza y Valenzuela, 2014, p. 257). Mientras que la capacidad gerencial es la habilidad para optimizar el logro de los objetivos planteados por la empresa (Ansoff y McDonell, 1997).

En este sentido, la capacidad de una empresa son las habilidades distintivas con respecto a otras empresas para el uso eficiente de los recursos que posee o que pueda adquirir, es decir, la capacidad de absorción para asimilar conocimiento. Por lo tanto, en una relación diádica las conexiones son basadas en la inversión de activos entre socios o relaciones sociales a partir de la confianza; donde a nivel de relaciones interorganizacionales, esta integración de recursos son considerados difíciles de identificar, entender e imitar, generando así ventajas competitivas (Dyer y Singh, 1998). Con ello, el desarrollo de capacidades en contextos determinados, basados en el conocimiento tácito, imperfectamente intransferibles, perdurables y costosos de imitar (Dyer y Singh, 1998; Lane y Lubatkin, 1998; Grant, 1991). De tal forma que el conocimiento de tipo colectivo o sectorial, a partir de la estrategia y aprendizaje interactivo, crea valor para las organizaciones y las capacidades gerenciales son clave al transferirlo, agregarlo y apropiarse para una ventaja competitiva sostenible (Spender, 1996; Grant, 1996). En otras palabras, las redes son un medio para adquirir conocimiento externo.

\section{ADQUISICIÓN DE CONOCIMIENTO EXTERNO EN LA INDUSTRIA ME- TALMECÁNICA: REDES Y GRUPOS ESTRATÉGICOS}

Las redes, desde el comportamiento organizacional, implican una dinámica de co-opetición y colaboración (Watkins y Bell, 2002) en un proceso de aprendizaje que parte de interacciones sociales (Brix, 2017; Knight, 2002; Nonaka et al. 2000) en el que se encuentran -clientes, proveedores, colegas, consumidores, empresas afiliadas, universidades, distribuidores, etc.- denominados los constituyentes externos (Nonaka et al., 2000) o stakeholders. Esta espiral del conocimiento -tácito y explícito- sobrepasa la frontera organizacional hacia comunidades de aprendizaje que trascienden 
a nivel departamental, divisional, sectorial y otras organizaciones, por medio de la socialización y entendimiento mutuo, basado en la confianza cuando se comparten experiencias e intercambiando conocimiento en el diálogo (Nonaka et al., 2000; Lane y Lubatkin, 1998; Dyer y Singh, 1998).

En estas prácticas, se hace referecia al concepto de una red interorganizacional (RIO), que se define como la integración de empresas en una red externa compuesta por todas las demás organizaciones (Ghoshal y Bartlett, 1990). Este fenómeno de estudio puede ser abordado a nivel de unidad empresarial o una unidad de red constituida por organizaciones (Gibb et al., 2017; Knight, 2002). Estas se abordan con respecto a las formas estructurales desde el comportamiento colaborativo -conectividad y comunidades-, en una representación dinámica de redes industriales, por ejemplo, en asociaciones tecnológicas y las relaciones para la creación de valor (Tatarynowicz et al., 2016). Este concepto aplicado a las PYMES se asocia con alianzas estratégicas, de innovación, desempeño y como facilitador de conocimiento (Stoian, Rialp y Dimitratos, 2017). No obstante, predominan los estudios enfocados a grandes empresas, a la EMN e internacionalizaciones (Ghoshal y Bartlett, 1990; Stoian et al., 2017).

El ambiente relacional (Sveiby, 2001; Nonaka 1994) se considera una vía para adquirir conocimiento, donde la diversidad de contactos facilita el acceso y aprendizaje para el desarrollo de nuevos productos, además de aumentar y compartir conocimiento en un entorno competitivo (Gibb et al., 2017; Bojica et al., 2012; Nonaka, 1994). Esto por medio de rutinas asociativas en el intercambio y transferencia de conocimiento, para contrarrestar costos transaccionales (Gibb et al., 2017). En adición, el conocimiento en redes y su influencia en las capacidades, permite a las empresas la eficiencia para la toma de decisiones al asociarse y diferenciar modelos divergentes de negocios entre las organizaciones, o bien, en industrias similares; ante la inercia en la adaptabilidad organizacional en entornos dinámicos (Sastry, 1997; Nonaka, 1994). Sin embargo, en una RIO, desde el proceso de gestión del conocimiento, se debe de considerar si los vínculos entre socios permiten el 
compartir, la transferencia y el ambiente de aprendizaje para la organización (Gibb et al., 2017) a partir de la captura de valor.

En la adquisición de conocimiento en una RIO intervienen diversos actores externos a la organización, representados por personas, empresas, gobiernos e instituciones sociales (Spender, 1996). Estos tipos de actores e instancias permiten ser medidos en una red social empresarial, a partir de la interdependencia entre organizaciones como es: la influencia y poder en la capacidad para adquirir información con socios potenciales de acuerdo a la posición de la red (Dyer y Singh, 1998) central o periférica que poseen, en relación a las empresas de un sector en un contexto dado. Esta influencia de actores o, bien, grupos sociales empresariales de acuerdo al nivel de formalidad es medida con base en la teoría de redes de tipo sociocéntrica; que consiste en los patrones de interacciones de individuos en una entidad social preexistente o una organización, a partir de un grupo de conexiones en una estructura social (Molina, 2005; Moreno, 1941). Lo anterior, con un enfoque sociológico de intercambio interpersonal y recíproco entre dos partes y la obtención de valor como son las transacciones económicas (Simmel, 1971).

Para este trabajo se hace referencia a dos tipos de grupos estratégicos para la competitividad de las organizaciones, uno formal y otro informal, ello, de acuerdo al objeto de su constitución. Las primeras corresponden a una asociatividad empresarial que pueden formar parte de un corporativo, vincularse vertical u horizontalmente, contratar servicios terciarios, fusionarse, crear otra nueva empresa o ser independientes entre sí, entre otros aspectos (González-Campo, 2010; Sánchez y Bracamonte, 2006; Domínguez, 2003; Pfeffer y Nowak, 1976). Pertenecen a los grupos de organizaciones legalmente autónomas, con un nivel alto de interdependencia, trabajo cooperativo, como son las redes estratégicas (Jarillo, 1988) y con fines de lucro (Knight, 2002; Jarillo, 1988).

Las segundas son redes sociales empresariales que se analizan con atributos a través de la visualización de interacciones en forma organizada y el comportamiento colectivo social organizativo en un lugar, región o espacio geográfico determinado (Molina, Ruiz y Teves, 2005; Van de Ven, 1976), independientemente de 
una estructura formal o en una organización económica industrial. Se refiere a un colectivo de organizaciones más libremente unidos, vinculados "por la proximidad geográfica, intereses o actividades similares, o la participación en la producción / entrega de un producto o servicio" (Knight, 2002, p. 430).

Con todo lo revisado, los aspectos de la evolución del sector, el planteamiento del problema y el objetivo de la presente investigación; a continuación se contrasta de manera empírica parte de los resultados de la investigación sobre la dinámica relacional en la adquisición de conocimiento por medio de una retícula. Se presenta una red social empresarial (Figura 3) para el caso de Juárez con la información proporcionada por las cuarenta empresas MIPYMES principalmente, sobre las actividades y acciones que tuvieron las empresas con otras empresas e instituciones durante el periodo de 2017-2018 (Estrada, 2020). Las empresas participantes se caracterizan por estar constituidas como personas físicas con actividad empresarial de la industria metalmecánica de capital nacional; empresas con registro en el INEGI-DENUE o ser empresas socias de la CANACINTRA, Clúster MACH y Clúster EGE. El cuestionario utilizado para el levantamiento de datos -Red interorganizacional para compartir conocimiento (Estrada, 2020)- incluye tres preguntas semiestructuradas sobre las RIO bajo los atributos: por nivel de contacto, influencia e intensidad. A su vez, estas se alinean con las variables de colaboración, transferencia y aprendizaje interorganizacional en el desarrollo de comunidades de práctica en un proceso de aprendizaje interactivo. Se definen como (Estrada, 2020):

- Interacción por contacto: se refiere a las demás empresas o instituciones con las que se ha integrado durante un periodo determinado y ha tenido contacto a través de acciones o actividades, con el fin de colaborar para intercambiar recursos o capacidades relacionados con la empresa.

- Interacción por influencia: se refiere a las demás empresas o instituciones con las que se ha integrado durante un periodo determinado, que considera haya tenido una influencia significativa para la empresa al momento de inter- 
cambiar información, como resultado de la comunicación constante en actividades relacionadas a la empresa.

- Interacción por intensidad: se refiere a las demás empresas o instituciones clave con las que se ha integrado durante un periodo determinado, que considera haya tenido un aprendizaje significativo para la empresa, derivado de relaciones de cooperación proactiva al compartir conocimiento entre un individuo, grupo de individuos o de instituciones.

Con los datos obtenidos de estos atributos se miden y analizan las conexiones entre las organizaciones con base en la teoría de redes. Para la representación del grafo se utiliza el Paquete igraph (Csardi y Nepusz, 2006), y en la realización de los cálculos estadísticos el Software R (R Core Team, 2019). Las interacciones son analizadas por medio de la cohesión interorganizacional (Rogers, 1974; Granovetter, 1973). A nivel micro, por medio de liderazgo "empresas centrales", involucramiento o reciprocidad, posición en la red -centralidad o marginalidad- (Gibb et al., 2017; Rogers, 1974; Granovetter, 1973), desde el ambiente de las organizaciones y el campo de la gestión del conocimiento. A nivel meso, los lazos fuertes y débiles por la aproximación o puentes en dichos lazo (Granovetter, 1973) en entornos dinámicos.

La Figura 3 considera datos agregados de los tres atributos medidos -contacto, influencia e intensidad-. En primer lugar, los resultados arrojan información que permite visualizar diversos actores clave que integran la red en la localidad, desde la influencia del conocimiento adquirido, entre ellos: empresas metalmecánicas (MM Metalmecánica); Academia (Academia); Clientes locales e internacionales (Clientes); Instituciones (Instituciones) financieras o gubernamentales a diferentes niveles; Otras empresas metalmecánicas (MM Otros) mencionadas por los participantes encuestados localidad e internacional; Otros sectores (Otros); Proveedores locales e internacionales (Proveedores); y Sociedades civiles (Instituciones).

En segundo lugar, se analiza la categorización de los actores y su sistema de interacciones. La red genera una comunidad de 237 

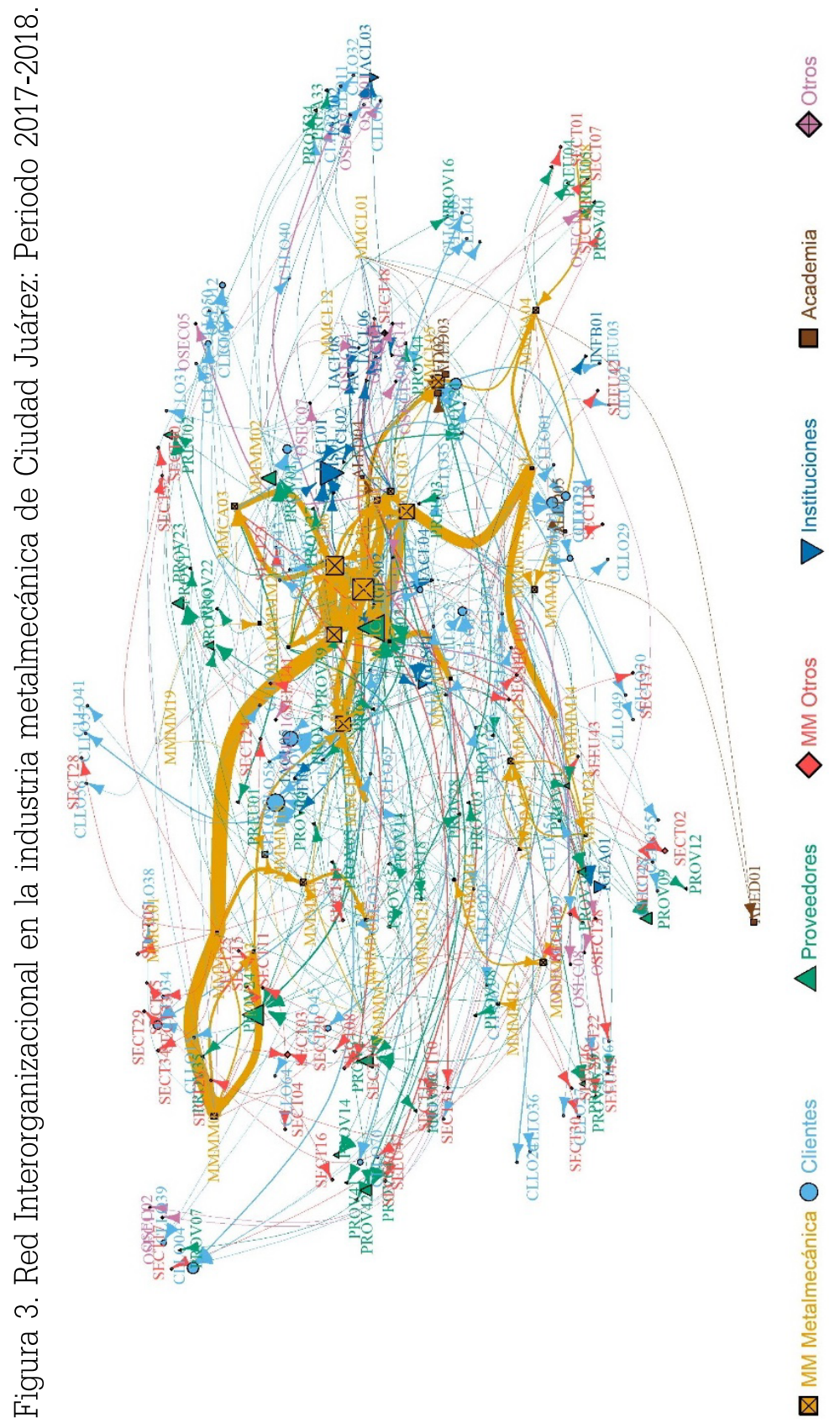

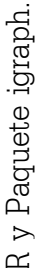

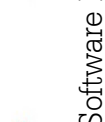

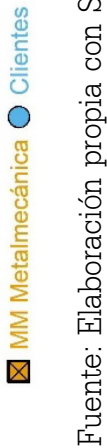


empresas -metalmecánicas con otros tipos de actores e instituciones-. Entre ellas, se establecen un total de 356 vínculos. Estos corresponden a empresas que se integran e interactúan con otras para la consulta de algún problema o implementación técnica, un requerimiento o renta de equipo de trabajo, o bien, para una mejor práctica de las empresas que aprenden, cuando estas colaboran, adquieren conocimiento por diversos canales de comunicación y aprenden de empresas maestras y de empresas centrales líderes del sector. Por lo tanto, las conexiones que se generan son dirigidas y dependerá de la reciprocidad para que la conexión sea bidireccional y de un aprendizaje mutuo.

A nivel micro se analiza el liderazgo de algunas empresas metalmecánicas consolidadas y proveedores clave a partir del núcleo-centro de la red. El núcleo lo constituyen empresas metalmecánicas con gran trayectoria en la localidad, principalmente una empresa con un poco más de 20 años en el mercado. Seguida de tres empresas con un promedio de más de 25 años en la localidad. En menor grado también lo integra una empresa emergente de reciente creación con menos de 5 años de establecida, a su vez, esta empresa lidera niveles altos de centralidad al ser una empresa con alto nivel de intermediación entre las empresas que conforman el núcleo con respecto al resto. Las cinco empresas comentadas forman parte del Clúster MACH. No obstante, la empresa con mayor número de menciones corresponde a un proveedor de la localidad. Este atributo lo posiciona como dentro de las empresas centrales influyentes en la industria metalmecánica y referente en la adquisición de conocimiento.

A nivel meso, la red en conjunto, tiene una alta intermediación entre las empresas centrales y los ubicados en la periferia de la red. En términos de comunidades de aprendizaje, las estrategias de asociatividad en grupos formalmente constituidos son evidentes en la red, ya que las empresas localizadas en el núcleo tienen un alto nivel de intermediación que les permite conectarse con empresas situadas en la periferia de la red. Por lo tanto, muestran una estructura de comunidad fuerte entre algunas de ellas con alta conectividad en la red y una influencia a nivel de región. 
Los lazos fuertes en términos de conocimiento se generan a partir de que las empresas centrales que cuentan con clientes potenciales y, a su vez, estos clientes complementan servicios con otras empresas del sector. Otro aspecto similar está en los proveedores con liderazgo en Ciudad Juárez.

Al respecto, la influencia de clientes y proveedores clave en la adquisición de conocimiento indica que, de cierta forma, se disemina un mismo tipo de conocimiento en la localidad y fungen como instituciones puente. En contraste, los lazos débiles que muestra el sector son los vínculos con la academia. Las empresas centrales tienen un mayor vínculo con instituciones académicas que el resto de las empresas participantes, por ejemplo: con la Universidad Tecnológica de Ciudad Juárez (UTCJ) y la Universidad Autónoma de Ciudad Juárez (UACJ), seguido del Centro de Bachillerato Tecnológico Industrial y de Servicios (CBTIS) y el Centro de Capacitación para el Trabajo Industrial (CECATI).

Por lo contrastado de manera empírica, el análisis de redes sociales empresariales basado en la gestión del conocimiento, independiente del nivel de formalidad, por asociatividad o grupos informales, permite percibir la realidad e influencia de actores clave en el proceso de un aprendizaje interactivo en la adquisición de conocimiento en la región de Juárez. La variable RIO permite abordar estudios del comportamiento organizacional hacia contextos externos de las empresas, donde las capacidades gerenciales para la captura y creación de valor son esenciales en la capacidad de absorción. La evidencia expuesta a través de una retícula para la industria metalmecánica con datos del año 2017-2018 indica que en la localidad se crean oportunidades de explorar conocimiento en grupos estratégicos, al igual que con clientes y proveedores clave, expuestos en el desarrollo de este análisis.

\section{DISCUSIÓN}

Examinar la evolución en las empresas metalmecánicas y el desarrollo de sus capacidades desde el paradigma del conocimiento y 
aprendizaje interactivo en comunidades y grupos estratégicos en un entorno meso y micro en Ciudad Juárez, permite la integración y análisis de evidencia científica; distingue los acontecimientos y determinantes a través del tiempo del sector; describe su composición con datos de fuentes oficiales como el Instituto Nacional de Estadística y Geografía (INEGI) y el DOF; e identifica, caracteriza y contrasta con los resultados de la investigación sobre la dinámica relacional en la adquisición de conocimiento por medio de una retícula.

El integrar y distinguir evidencia científica permite construir una línea de tiempo a partir de los enfoques económico, político y social para examinar los acontecimientos que activaron la frontera norte de México, y el desarrollo de la industria metalmecánica en Juárez. La realización de un segundo esquema afín al sector de estudio integra elementos asociados al conocimiento y capacidades a partir del Programa de la Industria Maquiladora, las generaciones de maquiladoras y el aprendizaje gradual de talleres y empresas de maquinados que fueron determinantes para la demanda y proliferación de un mayor número de empresas, derivado del desplazamiento hacia el exterior de personal especializado como son ingenieros y técnicos, entre otros. También el estudio proporcionó información a nivel de unidades de negocio que constituyen el sector para el año 2018 y 2021, mostrando un incremento en el número de pequeñas empresas y una disminución en las medianas empresas durante el 2021 con respecto al año 2018.

En complemento a la línea del tiempo, el estudio proporciona información sobre las prácticas de asociatividad empresarial en la región con los ejemplos del Clúster EGE y Clúster MACH. En el campo de estudio del comportamiento interorganizacional, se conciben dentro del aprendizaje interactivo como comunidades de práctica y grupos estratégicos en un entorno meso. El primer caso, el Clúster EGE desde la teoría de redes, se perciben como acciones emprendidas para generar lazos articulados entre sectores clave de la economía, por medio de una institución puente e intermediaria para la resolución de problemas emergentes con actores colectivos en una región (Granovetter, 1973), es decir, "sur- 
gen para encauzar el apoyo institucional, tecnológico y económico en el desarrollo de una cultura competitiva" (Casalet, 2000, p. 17) y fungen un papel en la prospección, planeación, aglomeración, articulación e intermediación y asistencia técnica con empresas similares o complementarias (De los Santos et al., 2017), con iniciativas en determinados sectores o cadenas productivas.

Mientras que el grupo empresarial Clúster MACH se considera un agente de enlace en las acciones estratégicas del sector, como entidad negociadora en aspectos económicos, tales como la gestión de recursos, costos transaccionales, conocimiento y tecnología (Cornejo et al., 2011). En otras palabras, empresas que se integran en una asociación que represente los intereses de la industria, promoviendo acciones de cooperación entre empresas del mismo sector o afines (Itami y Roehl, 1987); un colectivo de organizaciones libremente unidos y la vinculación "por la proximidad geográfica, intereses o actividades similares, o la participación en la producción/entrega de un producto o servicio" (Knight, 2002, p. 430).

Ambas asociaciones se enfocan en el modelo de la triple hélice al integrar esfuerzos entre la academia, industria y gobierno en un sistema industrial, desde la teoría económica e infraestructura del conocimiento (Etzkowitz y Leydesdorff, 1995). En el contexto de la teoría de la economía de la empresa representan nuevas entidades como asociaciones empresariales conformadas como grupo o alianzas estratégicas, integradas por empresas socias y homogéneas bajo un esquema de co-opetición en algunas actividades específicas (Pfeffer y Nowak, 1976; Domínguez, 2003). Este planteamiento alineado a una economía basada en el conocimiento, la competitividad y en un ambiente de clústeres industriales se deriva de la aproximación geográfica entre empresas, que permite la generación de acciones y prácticas de cooperación, coordinación, difusión de información y conocimientos heterogéneos y asimétricos para el aprendizaje y creación de conocimiento, promovido por una cultura social comunitaria de interacciones (Villavicencio, 2006; Maskell, 2001).

Evidencia científica (Casalet, 2000) confirma que en una sociedad surgen actores con estructuras informales, donde la confianza, 
la cultura organizacional de tipo relacional y la autocapacidad de organización permiten tener actividades coordinadas, toma de decisiones a nivel colectivo, así como el poder compartir información; donde las "asociaciones de empresas son organizaciones que pueden tener un impacto positivo en la dinámica económica de una región y en el desempeño de diversas industrias" (Cornejo et al., 2011, p. 111).

Estos elementos, contrastados con datos primarios, confirman que en el tema de redes sociales empresariales este estudio brinda información sobre los diferentes actores e instituciones que influyen para adquirir conocimiento alterno a las EMN -clientes del sector- como son proveedores y otras empresas del sector, tal como se muestra en la red. Algunas de estas empresas son clave y están localizadas en el núcleo-centro de la red por ser empresas consolidadas en la localidad. También, se destaca la influencia de un proveedor con un mayor grado de centralidad que las empresas metalmecánicas. No obstante, las empresas que conforman el núcleo, en primer lugar, son referentes para el desarrollo del aprendizaje y generación de conocimiento de otras empresas estudiadas ajenas al grupo estratégico Clúster MACH. También, el estudio permite identificar qué empresas registradas en el Clúster EGE tienen una posición estratégica y alta conectividad con el núcleo-centro de la red. Con estos elementos presentados, Bojica y colaboradores (2012) hacen referencia que las RIO permiten crear puentes sobre huecos estructurales evidentes en la red y oportunidades para conectar con nuevas relaciones directas por parte de las empresas del sector.

\section{CONCLUSIONES}

Durante el avance de este estudio se han presentado literatura y datos que soportan el desarrollo de la industria metalmecánica a partir de la influencia de la IME y las particularidades teóricas y empíricas sobre la adquisición de conocimiento en un ambiente de aprendizaje interactivo desde el enfoque relacional. La trayec- 
toria en la localidad ha forjado un desarrollo gradual de conocimientos especializados que constituyen y estructuran de manera organizativa las MIPYMES actuales. La fortaleza del estudio radica en que aporta evidencia sobre la existencia de otros agentes o empresas centrales, referentes para el aprendizaje, la adquisición de conocimiento y capacidades, como son proveedores del sector y empresas líderes de la misma industria, posicionadas estratégicamente a nivel meso y su influencia a nivel micro. Por lo tanto, el estudio contextualiza la dinámica relacional de la última década y los diversos tipos de actores involucrados, entre las oportunidades detectadas está el vincular en mayor medida lazos fuertes de las empresas con la academia.

En adición al tema de redes sociales empresariales, el estudio integra datos de empresas con gran liderazgo en la localidad; para futuras investigaciones, la interrogante que surge es saber si con otro tipo de muestra la influencia de las empresas líderes del núcleo-centro prevalecerían como un conjunto de empresas maestras que facilitan el aprendizaje o, bien, emergen otras nuevas a partir de grupos informales. En este sentido, una de las limitantes de la investigación es la existencia y acceso a una base de datos o padrón que integre las personas físicas con actividad empresarial dedicadas a este giro.

Ciudad Juárez es una zona fronteriza que cuenta con espacios laborales que han desarrollado conocimientos especializados e infraestructura afines a la proveeduría local de la IME a través de diferentes décadas; estos elementos, desde el enfoque de la gestión del conocimiento, se han dado por las habilidades y conocimientos explotados ante la necesidad de explorar y emprender negocios de proveeduría local. Por lo tanto, desde el campo del comportamiento interorganizacional, las empresas de la industria metalmecánica cuentan con ventajas competitivas sostenibles a nivel de región, a partir del fenómeno de prácticas de asociatividad formal o informal para de la adquisición de conocimientos y capacidades estratégicas, como vínculos estratégicos de conocimiento tácito, raro e imperfectamente imitable. De tal manera que se debe seguir explorando este tipo de estudios con la participa- 
ción de un mayor número de empresas y el análisis de redes en comunidades de aprendizaje.

Finalmente, se agradecen las aportaciones en el desarrollo de esta investigación al Dr. Alejandro Brugués de El Colegio de la Frontera Norte; Dr. Tomás Cuevas, Dr. Javier Chávez, Dra. Patricia Ramos, Dr. Francisco Bribiescas y Mtro. Miguel Ángel Calderón de la Universidad Autónoma de Ciudad Juárez; y Dr. Miguel Mujica de la Universidad de Carabobo.

\section{REFERENCIAS}

Abarca, Y. y Barreto, U. (2021). Capacidad de absorción del conocimiento, aprendizaje y tecnologías de la información en las organizaciones: estado del arte y evolución de la investigación. Apuntes Universitarios, 11(1), 95-122. doi:10.17162/ au.v11i1.558

Aiken, M. y Hage, J. (1968). Organizational Interdependence and Intra-Organizational Structure. American Sociological Review, 33(6), 912-930. http://www.jstor.org/stable/2092683

Ampudia, L. y De Fuentes, C. (2009). La industria de maquinados industriales en Querétaro y Ciudad Juárez. En G. Dutrénit (Coord.). Sistemas regionales de innovación: un espacio para el desarrollo de las Pymes. El caso de la industria de maquinados industriales (pp. 108-131). Universidad Autónoma Metropolitana.

Ansoff, H. I. y McDonell, E. J. (1997). La dirección estratégica en la práctica empresarial (2. ed.). Addison Wesley Longman.

Arellano, J. A. (2013). Proveedores a la Maquila. La relación de los Talleres de Maquinado Industrial y la Maquiladora en Ciudad Juárez, Chihuahua, a principio del Siglo xxı [tesis doctoral inédita]. Universidad Autónoma de Ciudad Juárez.Argueta, G. V. y Jiménez, C. P. (2017). Gestión del conocimiento. Innovar y patentar en empresas metalmecánicas en Ciudad Juárez, México. Chihuahua Hoy, 15(15), 257-288. http://erevistas.uacj. mx/ojs/index.php/ChihuahuaHoy/article/view/2042/1789 
Ávila, S. (2004). Chihuahua Siglo xxi: lessons learned in a decade of cluster-formation processes in Latin America. En T. Andersson, S. Schwaag-Serger, J. Sörvik y E. Wise. Cluster Policies Whitebook. IKED-International Organisation for Knowledge Economy and Enterprise Development (pp. 207-211). https:// portal.research.lu.se/portal/files/5954460/1304064.pdf

Bojica, A. M., Ruiz, M. y Fuentes, M. M. (2012). La adquisición de conocimiento a través de relaciones interorganizativas y la orientación emprendedora: el papel mediador del capital social de segundo orden. Cuadernos de Economía y Dirección de la Empresa, 15(3,), 141-153. doi: 10.1016/j.cede.2012.01.003

Brenner, T. (2000). The Evolution of Localised Industrial Clusters: Identifying the Processes of Self-organisation. Papers on Economics \& Evolution. Max-Planck Institute of Economics. http://www.mpiew-jena.mpg.de/files/2003/staff/brenner/selfaug.pdf

Brix, J. (2017). Exploring knowledge creation processes as a source of organizational learning: A longitudinal case study of a public innovation project. Scandinavian Journal of Management, 33(2),113-127. doi:10.1016/j.scaman.2017.05.001

Calderón, M. A. y Arenaza, A. (2015). El contexto social político y económico (1970-2000). Ciudad Juárez 1971-2000: de la ciudad tranquila a la transición global. En E. R. Staines Orozco y A. Y. Reyes Escalante (Coords.). Inventario de Monumentos Históricos y Edificios Relevantes de Ciudad Juárez. Tomo 3. Volumen 2 (pp. 23-28). Universidad Autónoma de Ciudad Juárez. Carrillo, J. y Gomis, R. (2005). Generaciones de maquiladoras. Un primer acercamiento a su medición. FRONTERA NORTE, 17(33), 25-51. http://www.scielo.org.mx/pdf/fn/ v17n33/v17n33a2.pdf

Carrillo, J. y Gomis, R. (2004). La maquiladora en datos: Resultados de una encuesta sobre tecnología y aprendizaje. El Colegio de la Frontera Norte.

Carrillo, J. y Matus, M. (2020). Will the Mexican Auto Parts Maquilas Be Able to Transit to I4.0? Paradigma económico, 12(2), 39-61. https://paradigmaeconomico.uaemex.mx/article/ view/14372/11438 
Casalet, M. (2000). Las redes institucionales en la creación del capital social. En J. Carrillo (Coord.). ¿Aglomeraciones locales o clusters globales? Evolución empresarial e institucional en el norte de México (pp. 17-43). El Colegio de la Frontera Norte/ Fundación Friedrich Ebert.

Casalet, M. y González, L. (2006). El entorno institucional y la formalización de las redes en el sector electrónico de Chihuahua. En D. Villavicencio (Coord.). La emergencia de dinámicas institucionales de apoyo a la industria maquiladora de México (pp. 49-87). Universidad Autónoma Metropolitana/Porrúa.

Centro de Competitividad Chihuahua. (15 de marzo de 2017). Inicio: Centro de Competitividad Chihuahua. Centro de Competitividad Chihuahua. http://cchcp.org.mx/2017/03/15/ nace-cluster-ege-para-potenciar-el-desarrollo-de-pymes-a-nivel-internacional/

Centro de Estudios Estratégicos ITESM (1993). Proyecto Chihuahua Siglo xxi. Primera Parte. Reporte Final-Tomo II. Centro de Estudios Estratégicos ITESM. https://repositorio.tec.mx/ handle/11285/574874

Cerutti, M. (2013). El algodón en el norte de México (1925-1965). De cultivo regional a materia prima estratégica. En M. Cerutti y A. Almaraz (Coords.). Algodón en el norte de México (19301970): impactos regionales de un cultivo estratégico (pp. 3772). El Colegio de la Frontera Norte.

Chandler, A. D. (1992). Organizational Capabilities and the Economic History of the Industrial Enterprise. Journal of Economic Perspectives, 6(3), 79-100. doi:10.1257/jep.6.3.79

Clúster de Empresas Globales Emergentes Chihuahua. (2019). Clúster EGE. Clúster Empresas Globales Emergentes Chihuahua. https://www.clusterege.com [Recuperado el 23 de septiembre de 2019].

Clúster de Manufactura Avanzada del Estado de Chihuahua. (15 de noviembre de 2018). Nosotros. Clúster de Manufactura Avanzada del Estado de Chihuahua. https://www.clustermach.com/\#nosotros 
Clúster de Manufactura Avanzada del Estado de Chihuahua. (2020). Acerca de nosotros. Expo-Automatización. Clúster de Manufactura Avanzada del Estado de Chihuahua. https://expo-automatizacion.com/acerca-del-evento/ [Recuperado el 14 de marzo de 2020].

Contreras, O. F., Carrillo, J. y Olea, J. (2012). Desprendimientos de las multinacionales, ¿una vía para el aprendizaje y la innovación en empresas locales? En J. Carrillo, A. Hualde y D. Villavicencio (Coords.). Dilemas de la innovación en México: Dinámicas sectoriales, territoriales e institucionales (pp. 301336). El Colegio de la Frontera Norte/Red Temática Complejidad, Ciencia y Sociedad de Conacyt.

Cornejo, E., Félix, J. y Ramírez, R. (2011). Asociaciones e innovación. Exploración de tres casos de asociaciones en clústers de la industria metalmecánica. En A. Martínez, A. García y P. L. López (Coords.). Innovación, transferencia tecnológica y políticas. Retos y oportunidades (pp. 109-136). CONCYTEG-UIACONACYT/Plaza y Valdés. https://www.researchgate.net/ profile/Adriana_Martinez_Martinez/publication/313662341_Innovacion_transferencia_tecnologica_y_politicas_Retos_y_ oportunidade/links/58a1f4e945851598babae64b/Innovaciontransferencia-tecnologica-y-politicas-Retos-y-oportunidade. pdf\#page $=109$

Csardi, G. y Nepusz, T. (2006). The igraph software package for complex network research. InterJournal, Complex Systems, 1965. http://igraph.org

Dawson, R. (2005). Developing Knowledge-based Client Relationships: Leadership in Professional Services (2. ${ }^{a}$ ed.). Elsevier

De los Santos, S., Carrillo, J., Plascencia, I., Villavicencio, D. y Esparza, I. (2017). Instituciones y actores para el impulso de la CTI en Chihuahua. En S. de los Santos, J. Carrillo, I. Plascencia, D. Villavicencio e I. Esparza, Ecosistemas de innovación en la frontera norte: Chihuahua y Coahuila (pp. 101-153). El Colegio de la Frontera Norte.

Desarrollo Económico del Estado de Chihuahua. A. C. (2018). Quienes Somos. Desarrollo Económico del Estado de Chihuahua. 
A. C. http://desec.org.mx/quienes-somos/ [Recuperado el 13 de diciembre de 2019].

Diario Oficial de la Federación. (15 de agosto de 1983). DECRETO para el fomento y operación de la industria maquiladora de exportación. Secretaría de Comercio y Fomento Industrial. Diario Oficinal de la Federación. http://www.dof.gob.mx/index_111.php?year=1983\&month=08\&day=15

Diario Oficial de la Federación. (13 de agosto de 1984). Programa Nacional de Fomento Industrial y Comercio Exterior 1984-1988. Secretaría de Comercio y Fomento Industrial. Diario Oficial de la Federación. http://dof.gob.mx/index. php?year $=1984 \&$ month $=08 \&$ day $=13$

Diario Oficial de la Federación [DOF]. (26 de Noviembre de 1986). DECRETO de Promulgación del Protocolo de Adhesión de México al Acuerdo General sobre Aranceles Aduaneros y Comercio. Secretaría de Relaciones Exteriores. México: Diario Oficinal de la Federación. Recuperado el 20 de 03 de 2019, de http:// http://dof.gob.mx/index.php?year=1986\&month=11\&day=26

Diario Oficial de la Federación . (20 de diciembre de 1993). Decreto de promulgación del Tratado de Libre Comercio de América del Norte. Secretaría de Relaciones Exteriores. Diario Oficinal de la Federación. http://www.dof.gob.mx/index. php?year $=1993 \&$ month $=12 \&$ day $=20$

Diario Oficial de la Federación. (1 de junio de 1998). DECRETO para el fomento y operación de la Industria Maquiladora de Exportación. Secretaría de Comercio y Fomento Industrial. Diario Oficinal de la Federación. http://dof.gob.mx/nota_detalle.php?codigo $=4880755 \&$ fecha $=01 / 06 / 1998$

Diario Oficial de la Federación. (01 de noviembre de 2006). DECRETO para el fomento y operación de la Industria Maquiladora de Exportación. Secretaría de Economía. Diario Oficinal de la Federación. http://www.dof.gob.mx/index_111. php?year $=2006 \&$ month $=11 \&$ day $=01$

Diario Oficial de la Federación. (21 de enero de 2015). Ley para el Desarrollo de la Competitividad de la Micro, Pequeña y Mediana Empresa. Secretaría de Economía. Diario Oficinal 
de la Federación. http://www.diputados.gob.mx/LeyesBiblio/ pdf/247_210115.pdf

Domínguez, M. D. (2003). Perspectivas teóricas de la cooperación y asociación para la competitividad empresarial en México. Aportes, VIII(22), 63-77. http://www.redalyc.org/ pdf/376/37602205.pdf

Donjuan, E. K. (2008). Capacidades de absorción dentro de clusters, el caso de los maquinados industriales de Ciudad Juárez [tesis de maestría inédita]. Universidad Autónoma de Ciudad Juárez..

Drucker, P. F. (2003). El management del futuro. Sudamericana.

--- (1999). El gran poder de las pequeñas ideas. Buenos Aires: Sudamericana.

Dutrénit, G. y Vera-Cruz, A. O. (2009). Derramas de conocimiento hacia instituciones. El caso de Ciudad Juárez. En G. Dutrénit (Coord.). Sistemas regionales de innovación: un espacio para el desarrollo de las Pymes. El caso de la industria de maquinados industriales (pp. 194-215). Universidad Autónoma Metropolitana.

Dyer, J. H. (2000). Collaborative Advantage: Winning Through Extended Enterprise Supplier Networks. Oxford University Press Dyer, J. H., y Singh, H. (1998). The Relational View: Cooperative Strategy and Sources of Interorganizational Competitive Advantage. The Academy of Management Review, 23(4), 660679. doi:10.2307/259056

Dyer, J. H., Singh, H., y Hesterly, W. S. (2018). The relational view revisited: A dynamic perspective on value creation and value capture. Strategic Management Journal, 39(12), 3140-3162. doi: 10.1002/smj.2785

El Colegio de la Frontera Norte. (2002). Encuesta Aprendizaje Tecnológico y Escalamiento Industrial en Plantas Maquiladoras. Departamento de Estudios Sociales, El Colegio de la Frontera Norte.Estrada, B. (2020). Colaboración y red interorganizacional para el desempeño, mediante la capacidad de absorción, aprendizaje y transferencia de conocimiento en el sector de la industria metalmecánica en Ciudad Juárez [tesis doctoral inédita]. Universidad Autónoma de Ciudad Juárez. 
Etzkowitz, H. y Leydesdorff, L. (1995). The Triple Helix-UniversityIndustry-Government Relations: A Laboratory for Knowledge Based Economic Development. EASST Review, 14(1), 14-19. https://papers.ssm.com/sol3/papers.cfm?abstract_id=2480085

Fahy, K. M., Easterby-Smith, M. y Lervik, J. E. (2013). The Power of Spatial and Temporal Orderings in Organizational Learning. Management Learning, 45(2), 123-144. doi:10.1177/1350507612471925

Fuentes, C. M. y Fuentes, N. A. (2004). Desarrollo económico en la frontera norte de México: de las políticas nacionales de fomento económico a las estrategias de desarrollo económico local. Araucaria, 5(11), 71-86. https://revistascientificas.us.es/ index.php/araucaria/article/view/1032/936

Fuentes, N. A., Ruiz, W., González, G. y Brugués, A. (2016). Exploración de los impactos de la homologación del IVA en la región y franja fronteriza, con particular referencia al caso de Baja California. El Cotidiano, (195), 63-74. https://www. redalyc.org/pdf/325/32543454008.pdf

Ghoshal, S. y Bartlett, C. A. (1990). The Multinational Corporation as an Interorganizational Network. Academy of Management Review, 15(4), pp. 603-624.

Gibb, J., Sune, A. y Albers, S. (2017). Network learning: Episodes of interorganizational learning towards a collective performance goal. European Management Journal, 35(1), 15-25. doi:10.1016/j.emj.2016.09.001

Goerzen, A. (2007). Alliance Networks and Firm Performance: The Impact of Repeated Partnerships. Strategic Management Journal, 28(5), 487-509. doi:10.1002/smj.588

González-Campo, C. H. (2010). Interacción teórica para la caracterización de redes empresariales. Innovar, 20(37), 117-132. https://www.redalyc.org/pdf/818/81818989010.pdf

González, E. L. y Barajas, M. R. (2004). Los procesos de aprendizaje en la industria electrónica maquiladora. ¿Una senda predefinida? En J. Carrillo y R. Partida (Coords.). La Industria Maquiladora Mexicana: aprendizajes tecnológicos, impactos 
regionales y entornos institucionales (pp. 19-67). El Colegio de la Frontera Norte/Universidad de Guadalajara.

Granovetter, M.S. (1973). The Strength of Weak Ties. American Journal of Sociology, 78(6), 1360-1380. doi:10.1086/225469

Grant, R. M. (1991). The Resource-Based Theory of Competitive Advantage: Implications for Strategy Formulation. California Management Review, 33(3), 114-135.

Grant, R. M. (1996). Toward a Knowledge-Based Theory of the Firm. Strategic Management Journal, 17, 109-122.

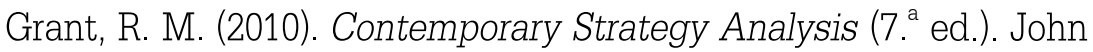
Wiley \& Sons Ltd.

Hayek, F. A. (1937). Economics and Knowledge. Economica, 4(13), 33-54. doi: $10.2307 / 2548786$

Hayek, F. A. (1945). The use of knowledge in society. The American Economic Review, 35(4), 519-530. DOI: 10.1142/9789812701275_0025.

Hualde, A. (2006). Claroscuros del aprendizaje en la maquiladora de la frontera norte: empresa, mercado de trabajo y territorio. En D. Villavicencio (Coord.). La emergencia de dinámicas institucionales de apoyo a la industria maquiladora de México (pp. 145-183). Universidad Autónoma Metropolitana/Porrúa.

IA. Center. (2021). Quienes somos. IA. Center. https://www. ia.center/es/quienes-somos/ [Recuperado el 2 de marzo de 2021].

Instituto de Apoyo al Desarrollo Tecnológico. (2018). CENALTEC Juárez: Instituto de Apoyo al Desarrollo Tecnológico. https:// www.inadet.com.mx/Home/juarez/ [Recuperado el 18 de julio de 2019].

Instituto Nacional de Estadística y Geografía. (9 de octubre de 2018a). Directorio Estadístico Nacional de Unidades Económicas (DENUE). Instituto Nacional de Estadística y Geografía. http://www3.inegi.org.mx/sistemas/mapa/denue/default.aspx Instituto Nacional de Estadística y Geografía. (14 de noviembre de 2018b). Industria Manufacturera. Instituto Nacional de Estadística y Geografía. http://www.beta.inegi.org.mx/temas/ manufacturas/ 
Instituto Nacional de Estadística y Geografía. (14 de marzo de 2021). Directorio Estadístico Nacional de Unidades Económicas (DENUE). Instituto Nacional de Estadística y Geografía. http://www3.inegi.org.mx/sistemas/mapa/denue/default.aspx Itami, H. y Roehl, T. W. (1987). Mobilizing Invisible Assets. Harvard University Press.

Jarillo, J. C. (1988). On Strategic Networks. Strategic Management Journal, 9(1), 31-41.

Jiménez, C. P., Argueta, G. V. y Leiner, M. (2017). Impacto del Nivel Educativo en la Visión Estratégica de Empresarios Metalmecánicos de Ciudad Juárez. En V. Hernández, E. Galeana, M. A. Valenzo y P. Chávez (Comps.). Estudios Organizacionales en las Ciencias Administrativas ante los Retos del Siglo XXI (pp. 2881-2901). Universidad Michoacana de San Nicolás de Hidalgo.

Jiménez, R. y Sánchez, G. (2015). Capital Social y desempeño empresarial: la industria metalmecánica en Ciudad Juárez, México. Estudios Regionales en Economía, Población y Desarrollo. Cuadernos de Trabajo de la UACJ, 26, 3-33. http:// erevistas.uacj.mx/ojs/index.php/estudiosregionales/article/ view/1543/1361

Katz, J. (1982). Cambio Tecnológico en la Industria Metalmecánica Latinoamericana. Resultado de un Programa de Estudios de Caso. Banco Interamericano de Desarrollo/Comisión Económica para América Latina/Centro Internacional de Investigaciones para el Desarrollo/Programa de las Naciones Unidas para el Desarrollo. https://publications.iadb.org/bitstream/ handle/11319/4153/Cambio_tecnológico_en_la_industria_metalmecánica_latinoamericana\%2C_resultados_de_un_programa_de_estudios_de_casos.pdf?sequence=1

Knight, L. (2002). Network learning: Exploring learning by interorganizational networks. Human Relations, 55(4), 427-454. doi:10.1177/0018726702554003

Lane, P. J. y Lubatkin, M. (1998). Relative Absorptive Capacity and Interorganizational Learning. Strategic Management Journal, 
19, 461-477. doi: 10.1002/(SICI)1097-0266(199805)19:5<461::AIDSMJ953>3.0.CO;2-L

Lawson, B., Petersen, K. J., Cousins, P. D. y Handfield, R. B. (2009). Knowledge Sharing in Interorganizational Product Development Teams: The Effect of Formal and Informal Socialization Mechanisms. Journal of Product Innovation Management, 26(2), 156-172. doi:10.1111/j.1540-5885.2009.00343.x

Manuj, A. O. y Yazdanparast, A. (2013). The Quest for Competitive Advantage in Global Supply Chains: The Role of Interorganizational Learning. Transportation Journal, 52(4), 463-492. doi:10.5325/transportationj.52.4.0463

Maskell, P. (2001). Towards a Knowledge-Based Theory of the Geographical Cluster. Industrial and Corporate Change, 10(4), 921-943. Recuperado el 08 de 08 de 2019, de https://pdfs. semanticscholar.org/d123/9fd0da18a1e00e45440e96d1afdeab3 b04bb.pdf

Mendoza, E. (1982). Historia de los programas federales para el desarrollo económico de la frontera norte. En M. Ojeda (Comp.). Administración del Desarrollo de la Frontera Norte. El Colegio de México.

Mendoza, J. G. y Valenzuela, A. (2014). Aprendizaje, innovación y gestión tecnológica en la pequeña empresa. Un estudio de las industrias metalmecánica y de tecnologías de información en Sonora. Contaduría y Administración, 59(4), 253-284. http:// www.scielo.org.mx/pdf/cya/v59n4/v59n4a11.pdf

Menolli, A., Reinehr, S. y Malucelli, A. (2013). Organizational Learning Applied to Software Engineering: A Systematic Review. International Journal of Software Engineering and Knowledge Engineering, 23(8), 1153-1175. doi:10.1142/S0218194013500356

Molina, J. L. (2005). El estudio de las redes personales: contribuciones, métodos y perspectivas. EMPIRIA. Revista de Metodología de Ciencias Sociales, 10, 71-105. http://www.redalyc. org/articulo. oa?id=297123998003

Molina, J. L., Ruiz, A. A. y Teves, L. (2005). Localizando geográficamente las redes personales. Redes: revista hispana para el análisis de redes sociales, 8(5), 1-21. 
Morales, J. (1999). La política regional en Chihuahua: el programa Chihuahua Siglo xxi. Avances n. 9. Cuadernos de Trabajo de la Coordinación de Investigación y Posgrado en el ICSA. Universidad Autónoma de Ciudad Juárez, Ciudad Juárez.Moreno, J. L. (1941). Foundations of Sociometry: An Introduction. Sociometry, 4(1), 15-35. doi: 10.2307/2785363

Nodari, F., Oliveira, M. y Gastaud, A. C. (2016). Organizational Performance through the Donation and Collection of Interorganizational Knowledge. VINE Journal of Information and Knowledge Management Systems, 46(1), 85-103. doi:10.1108/ VJIKMS-08-2014-0052

Nonaka, I. (1994). A Dynamic Theory of Organizational Knowledge Creation. Organization Science, 5(1), 14-37. https://www.jstor. org/stable/pdf/2635068.pdf

Nonaka, I., Toyama, R. y Konno, N. (2000). SECI, Ba and Leadership: a Unified Model of Dynamic Knowledge Creation. Long Range Planning, 33(1), 5-34. doi:10.1016/S0024-6301(99)00115-6 Pfeffer, J. y Nowak, P. (1976). Joint Ventures and Interorganizational Interdependence. Administrative Science Quarterly, 21(3), 398-418. doi:10.2307/2391851

Quezada-Torres, W. D., Hernández-Pérez, G. D., González-Suárez, E., Comas-Rodríguez, R., Quezada-Moreno, W. F. y MolinaBorja, F. (2018). Gestión de la tecnología y su proceso de transferencia en Pequeñas y Medianas Empresas metalmecánicas del Ecuador. Ingeniería Industrial, 39(3), 303-314. http:// scielo.sld.cu/pdf/rii/v39n3/1815-5936-rii-39-03-303.pdf

R Core Team. (2019). R: A language and environment for statistical computing. R Foundation for Statistical Computing. https:// www.R-project.org/. [Recuperado el 2 de octubre de 2018]. Rogers, D. L. (1974). Sociometric Analysis of Interorganizational Relations: Application of Theory and Measurement. Rural Sociology, 39(4), 487-503.

Sánchez, J. M. y Bracamonte, Á. (2006). Aglomeraciones industriales y desarrollo económico: El caso de Hermosillo, 1998. FRONTERA NORTE, 18(36), 87-124. doi:10.17428/rfn.v18i36.1028 
Sastry, M. A. (1997). Problems and Paradoxes in a Model of Punctuated Organizational Change. Administrative Science Quarterly, 42(2), 237-27. doi:10.2307/2393920

Secretaría de Economía. (2012). Sexto Informe de Labores 20062012. Secretaría de Economía. http://www.economia.gob.mx/ files/6_informe.pdf

Secretaría de Programación y Presupuesto y Nacional Financiera. (1982). La Industria Metalmecánica y de Bienes de Capital en México. INEGI

Simmel, G. (1971). On individuality and social forms. University of Chicago Press.

Spender, J. C. (1996). Making knowledge the basis of a dynamic theory of the firm. Strategic Management Journal, 17(Winter Special Issue), 45-62. doi:10.1002/smj.4250171106

Stoian, M.C., Rialp, J. y Dimitratos, P. (2017). SME Networks and International Performance: Unveiling the Significance of Foreign Market Entry Mode. Journal of Small Business Management, 55(1), pp. 128-148. doi:10.1111/jsbm.12241

Sveiby, K. E. (2001). A knowledge-based theory of the firm to guide in strategy formulation. Journal of Intellectual Capital, 2(4), 344-358. doi:10.1108/14691930110409651

Tatarynowicz, A., Sytch, M. y Gulati, R. (2016). Environmental Demands and the Emergence of Social Structure: Technological Dynamism and Interorganizational Network Forms. Administrative Science Quarterly, 61(1), 52-86. doi:10.1504/ IJESB.2015.067286

Timmons, W. H. (1990). El Paso. A Borderlands History. Texas Wester Press.

Van de Ven, A. H. (1976). On the Nature, Formation, and Maintenance of Relations among Organizations. The Academy of Management Review, 1(4), 4-36. doi:10.5465/amr.1976.4396447 Vázquez, A. (1999). Desarrollo, redes e innovación. Lecciones sobre el desarrollo endógeno. Pirámide.

Vera-Cruz, A. O. y Dutrénit, G. (2004). La IED y las capacidades de innovación y desarrollo locales: lecciones del estudio de los casos de la maquila automotriz y electrónica en Ciudad de 
Juárez (No. 25668). Naciones Unidas I Comisión Económica para América Latina y el Caribe (CEPAL). https://repositorio. cepal.org/handle/11362/25668

Vera-Cruz, A. O. y Dutrénit, G. (2009). Derramas de las ETN a través de la movilidad de los trabajadores. Evidencia de pymes de maquinados en Ciudad Juárez. En G. Dutrénit (Coord.). Sistemas regionales de innovación: un espacio para el desarrollo de las Pymes. El caso de la industria de maquinados industriales (pp. 172-193). Universidad Autónoma Metropolitana.

Vera-Cruz, A. O. y Gil, J. L. (2006). Creación de redes como un mecanismo para el desarrollo de capacidades de los proveedores mexicanos de la maquila: el caso de la industria del maquinado. En D. Villavicencio (Coord.). La emergencia de dinámicas institucionales de apoyo a la industria maquiladora de México (pp. 185-206). Universidad Autónoma Metropolitana.

Villavicencio, D. (2006). El surgimiento de un entorno institucional de apoyo a las empresas maquiladoras en la frontera norte de México. En D. Villavicencio (Coord.). La emergencia de dinámicas institucionales de apoyo a la industria maquiladora de México (pp. 17-48). Universidad Autónoma Metropolitana.

Watkins, M., y Bell, B. (2002). The experience of forming business relationships in tourism. International Journal of Tourism Research, 4(1), 15-28. doi:10.1002/jtr.337 